\title{
Pretratamientos de deshidratación osmótica para mejorar el color y prevenir las pérdidas de antocianinas y de antioxidantes en dulces de frutilla
}

\author{
LM. Rodoni ${ }^{1,2}$, ML Lemoine ${ }^{1,2}$, AR Vicente ${ }^{1,2}$ \\ ${ }^{1}$ CIDCA, CONICET-UNLP. La Plata, Bs.As. . ${ }^{2}$ LIPA, FCAyF, UNLP. La Plata, Bs.As. \\ E-mail: luisrodoni@gmail.com
}

\section{Resumen}

Recientemente ha aumentado el interés en la búsqueda de métodos de procesamiento que permitan preservar compuestos bioactivos presentes en los frutos. La deshidratación osmótica (DO) es un proceso que extrae parte del agua y solutos de los alimentos por intercambio con una solución altamente concentrada de un osmolito. Se estudió la influencia de pretratamientos de DO sobre la calidad y nivel de compuestos bioactivos en dulce de frutilla. Frutillas $(80 \%$ de color superficial rojo) se cortaron longitudinalmente, empleándose una mitad para la elaboración con D0 y tratamiento térmico y la restante para una elaboración convencional. Para los dulces con DO previa, las mitades fueron cortadas en trozos de $3 \mathrm{~mm}$, a los cuales se les adicionó $60 \%$ de sacarosa, dicha mezcla reposó por $15 \mathrm{~min}$ a $20^{\circ} \mathrm{C}$. Los frutos deshidratados (FD) $(22 \% \pm 2$ de sólidos solubles (SS)) y el jarabe obtenido (SS $=63 \% \pm 3$ ) se separaron y el jarabe fue concentrado (JC) hasta $81 \pm 3 \%$ SS. Los FD fueron procesados y adicionados al JC, obteniéndose una mezcla de $54 \pm 2 \%$ de SS la cual se evaporó hasta $64 \pm 2 \%$ de SS $(n=7)$. Paralelamente, se realizaron elaboraciones tradicionales mezclando frutillas con $60 \%$ de sacarosa $(S S=44 \% \pm 2$ ) y evaporando hasta $64 \pm 2 \%$ de SS $(n=7)$. Se evaluó el color $\left(L^{*}, a^{*}\right.$ y $b^{*}$, 'Hue, y chroma), antocianinas y capacidad antioxidante por ABTS ${ }^{\bullet+}$. La D0 redujo el tiempo de tratamiento térmico. Los dulces con D0 fueron más claros (mayor $L^{*}$ ) que los control. Los valores de $a^{*}$ y $b^{*}$ fueron más elevados, indicando menor pérdida de tonos rojos y amarillos. Los dulces con D0 tuvieron un 20\% más de antocianinas y un $11 \%$ más de poder antioxidante que los tradicionales. Esta técnica permitió obtener dulces con un color más natural, con incremento en compuestos bioactivos y antioxidantes. Esta información puede resultar útil para la industria de confituras.

Palabras clave: mermelada, evaporación, azúcar, bioactivos

\section{Introducción}

Las frutillas son apreciadas por los consumidores debido a sus características de sabor, aroma y color. Las frutillas pueden consumirse frescas 0 procesadas de diferentes formas dentro de las que se destaca la obtención de confituras. El color característico de las frutillas está determinado por su contenido en antocianinas, compuestos de naturaleza fenólica (flavonoides) generalmente unidos covalentemente a un azúcar (Goulas et al., 2012). En frutilla la antocianina predominante es la pelargonidina3-glucósido (Wang y Lewers, 2007). Además de su rol en el color, las antocianinas son importantes compuestos bioactivos con reconocida actividad antioxidante. Estos compuestos son sensibles al calor (Howard et al., 2012), por lo que pueden verse afectados durante la elaboración de las confituras.

Varios trabajos han evaluado la influencia de factores de proceso tales como el tipo de pectina utilizado, la 
temperatura de cocción (Holzwarth et al., 2013), el estado de madurez de los frutos (Levaj et al., 2010), el reemplazo de azúcares por edulcorantes (Basu et al., 2012) 0 el agregado de diferentes agentes gelificantes (Javanmard et al., 2012) en la calidad de confituras. En la mayor parte de los trabajos se ha observado una reducción de los niveles de antioxidantes (Rababah et al., 2012). De todos modos, se ha sugerido que la hidrólisis de los azúcares asociados con los antioxidantes podría aumentar su biodisponibilidad en ciertos casos (Rickman et al., 2007). La deshidratación osmótica (D0) es un proceso de concentración que consiste en extraer parte del agua de los alimentos por intercambio con una solución altamente concentrada de un osmolito sin necesidad de recurrir a tratamientos con alta temperatura (Jain et al., 2010). Si bien a priori la D0 puede ser utilizada por sí sola para la preparación de mermeladas, debido a las bajas temperaturas utilizadas, se ve comprometida la estabilidad microbiológica y enzimática del producto durante el almacenamiento (Igual et al., 2013). No obstante, la D0 podría ser útil en forma previa a la cocción para reducir el procesado térmico y preservar mejor las propiedades nutricionales y sensoriales de los frutos. En ese sentido, el objetivo de este trabajo fue evaluar la influencia de pretratamientos con deshidratación osmótica sobre el color, el contenido de antocianinas y la capacidad antioxidante de dulces de frutillas.

\section{Materiales y métodos}

\subsection{Material vegetal}

Se utilizaron frutillas (Fragaria $\mathrm{x}$ ananassa) con $80 \%$ de color superficial rojo y $8,3 \%$ de sólidos solubles, las cuales, luego de ser lavadas con agua conteniendo $100 \mathrm{ppm}$ de $\mathrm{HClO}$, fueron desprovistas del cáliz y cortadas longitudinalmente a la mitad. Una mitad se utilizó para la elaboración de dulce con D0 previa y la otra para la elaboración tradicional (control).

\subsection{Elaboración de dulce}

Las etapas del proceso de elaboración se encuentran resumidas en la Figura 1. Para la elaboración de dulces con deshidratación osmótica previa (D0), las frutillas procesadas como se indica en la sección 2.1 fueron nuevamente cortadas transversalmente en trozos de 3 $\mathrm{mm}$ de espesor y a las mismas se les adicionó $60 \%$ p/p de sacarosa. La mezcla se mantuvo 15 min a $20^{\circ} \mathrm{C}$ para permitir un proceso de deshidratación previa. Posteriormente los frutos deshidratados se separaron (FD) (SS $=22 \% \pm 2)$, del jarabe obtenido por la deshidratación (J); $(S S=63 \% \pm 3)$ y este último fue concentrado hasta $81 \% \pm 3$ de SS. Los FD fueron procesados y se adicionó al jarabe concentrado (JC), obteniéndose una mezcla de $54 \% \pm 2$ de SS la cual se evaporó hasta $64 \% \pm 2$ de SS. Para las elaboraciones control se mezclaron los frutos trozados como se indicó en 2.1 , se adicionó $60 \%$ de sacarosa ( $S S=44 \% \pm 2$ ) y se evaporó hasta $64 \% \pm 2$ de SS. El ensayo siguió un diseño en bloques donde cada bloque representa una elaboración DO y otra tradicional. Se realizaron 7 elaboraciones de cada tipo de dulce.

\subsection{Color}

Se utilizó un colorímetro (Minolta, Modelo CR-400) provisto con un cabezal de inmersión. Se midieron las variables de color CIE a*, b* y L* y se calculó el Hue (ángulo de color) y el croma $\left(C^{*}\right)$ utilizando las siguientes ecuaciones:

$$
\begin{gathered}
\text { Hue }=\operatorname{atan}\left(b^{*} / a^{*}\right) \\
c^{*}=\left(\left(a^{*}\right)^{2}+\left(b^{*}\right)^{2}\right)^{1 / 2}
\end{gathered}
$$

\subsection{Antocianinas}

Aproximadamente $2 \mathrm{~g}$ de dulce de frutilla fueron extraídos con $20 \mathrm{~mL}$ de etanol frío al $75 \% \mathrm{v} / \mathrm{v}$ conteniendo $0.1 \% \mathrm{p} / \mathrm{p}$ de HCl. La mezcla se centrifugó a 9.000 × $g$ por 10 min a 4 ${ }^{\circ} \mathrm{C}$. Se tomaron $5 \mathrm{~mL}$ del sobrenadante y se llevaron a 10 $\mathrm{mL}$ con el solvente de extracción. Se medió la absorbancia a $515 \mathrm{~nm}$. En contenido de antocianinas se expresó como mg de pelargonidina-3-glucósido equivalente por kg de peso fresco usando el coeficiente de extinción $\varepsilon=36.000 \mathrm{M}^{-1}$ $\mathrm{cm}^{-1}$.

\subsection{Antioxidantes por ABTS ${ }^{\bullet+}$}

Aproximadamente $2 \mathrm{~g}$ de dulce de frutilla fueron extraídos con $20 \mathrm{~mL}$ de etanol. La mezcla se centrifugó a $9.000 \times \mathrm{g}$ por $10 \mathrm{~min}$ a $4{ }^{\circ} \mathrm{C}$. Se tomaron $5 \mathrm{~mL}$ del sobrenadante y se llevaron a $10 \mathrm{~mL}$ con etanol. El sobrenadante se utilizó para la determinación de antioxidantes por el radical ABTS ${ }^{\cdot+}$. A 1 $\mathrm{mL}$ de solución del radical ABTS ${ }^{+}+(7 \mathrm{mM}$ de ABTS con 2,45 $\mathrm{mM} \mathrm{K}_{2} \mathrm{~S}_{2} \mathrm{O}_{8}$, absorbancia $0,700+/-0,05$ a $734 \mathrm{~nm}$ ) se le adicionaron $25 \mu \mathrm{L}$ del extracto. La mezcla se dejó reaccionar 6 minutos y se leyó la absorbancia a $734 \mathrm{~nm}$. Los resultados se expresaron como $\mathrm{mg}$ de Trolox por $\mathrm{kg}$ de peso fresco. Se realizaron tres replicados por cada tratamiento y tiempo de 

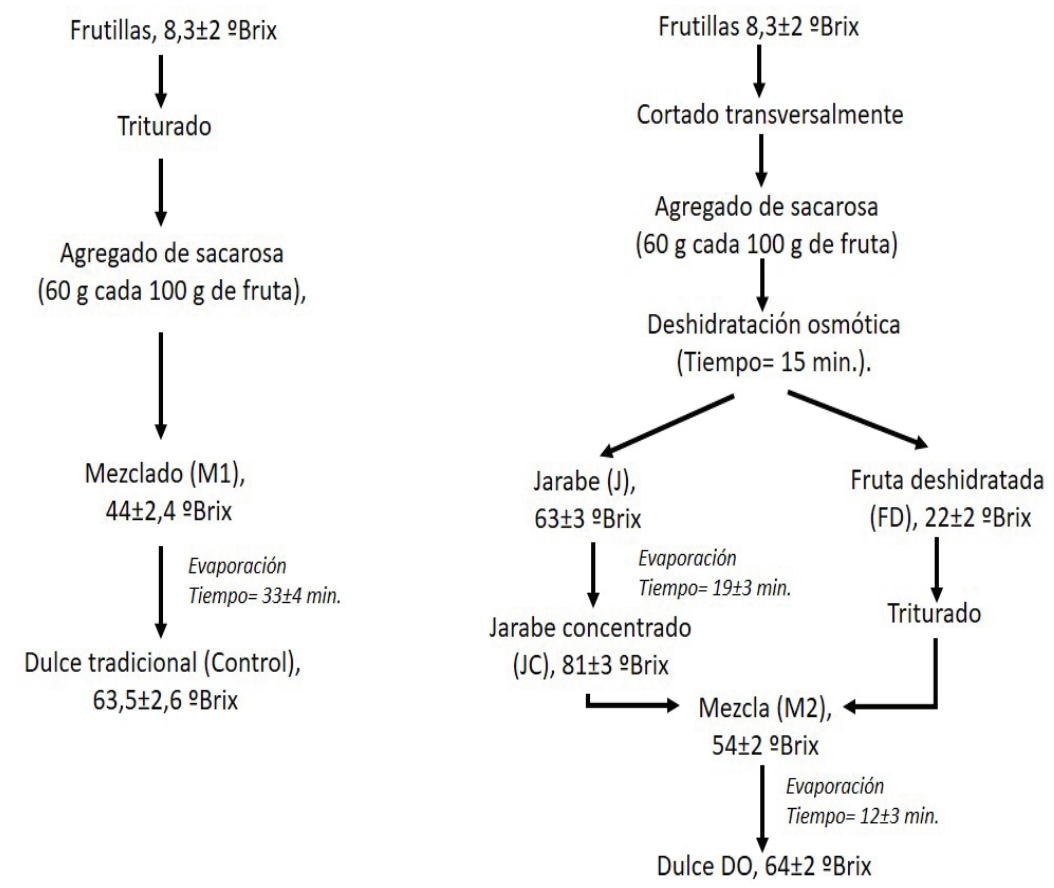

Figura 1: Esquema de las elaboraciones para los dulces de frutilla; izquierda, elaboración tradicional (control); derecha, elaboración con deshidratación osmótica previa. Se muestran promedios y desviaciones en algunos parámetros obtenidos considerando todas las elaboraciones. $n=7$.

almacenamiento.

2.6. Análisis estadístico

Los datos fueron analizados mediante un ANOVA. Se utilizó

\section{Resultados y discusión}

\subsection{Color}

En la Figura 2 pueden observarse la apariencia y los parámetros de color para ambos tipos de dulce, control y D0. Los dulces con D0 previa tuvieron un color más semejante al fruto fresco en comparación a los dulces control (Figuras $2 \mathrm{~A}$ y $2 \mathrm{~B}$ ). La luminosidad ( $\mathrm{L}^{*}$ ) para los dulces control fue de 21 , mientras que en los dulces con D0 previa fue de 22,8 (Figura 2C). El mayor $L^{*}$ de los dulces D0 en comparación a los dulces control puede estar relacionado a un menor contenido de compuestos pardos derivados de la reacción de Maillard (Flores y del Castillo, 2016) o de la degradación térmica de antocianinas (Jackman et al., 1987). Los valores de $a^{*}$ y $b *$ fueron de 20,1 y 9,2 respectivamente en dulces D0 mientras que en los tradicionales fue inferior, aproximadamente 15,8 y 6,1 para $a *$ y $b$ * respectivamente un diseño en bloque donde cada bloque representó una elaboración control y una con D0 previa, las medias se compararon empleando un test de Tukey, $\alpha \leq 0,05$.

(Tabla 1). Estos parámetros derivaron en un ${ }^{\circ}$ Hue de 24,5 , mientras que en los dulces tradicionales el ${ }^{\circ}$ Hue fue de 21 (Figura 2D). El menor ${ }^{\circ}$ Hue en los dulces control podría deberse a que la fruta sufrió mayor tiempo de exposición a altas temperaturas. Con respecto a la saturación del color $\left(C^{*}\right)$, los dulces D0 tuvieron un $C^{*}$ de 22,1 mientras que en los controles fue de 16,9 (Figura 2E). Los mayores valores del $C^{*}$ de los dulces DO en relación a los controles puede estar relacionado a un mayor contenido de antocianinas, y pueden tomarse como referencia de los cambios del color en dulces de frutilla (Watanabe et al., 2011). Adicionalmente, los dulces D0 presentaron un aroma y sabor más cercano al fruto fresco que los controles (datos no mostrados). En síntesis, el pretratamiento de D0 antes de la cocción permitió obtener un dulce con características de color diferentes a las obtenidas en las elaboraciones tradicionales. 


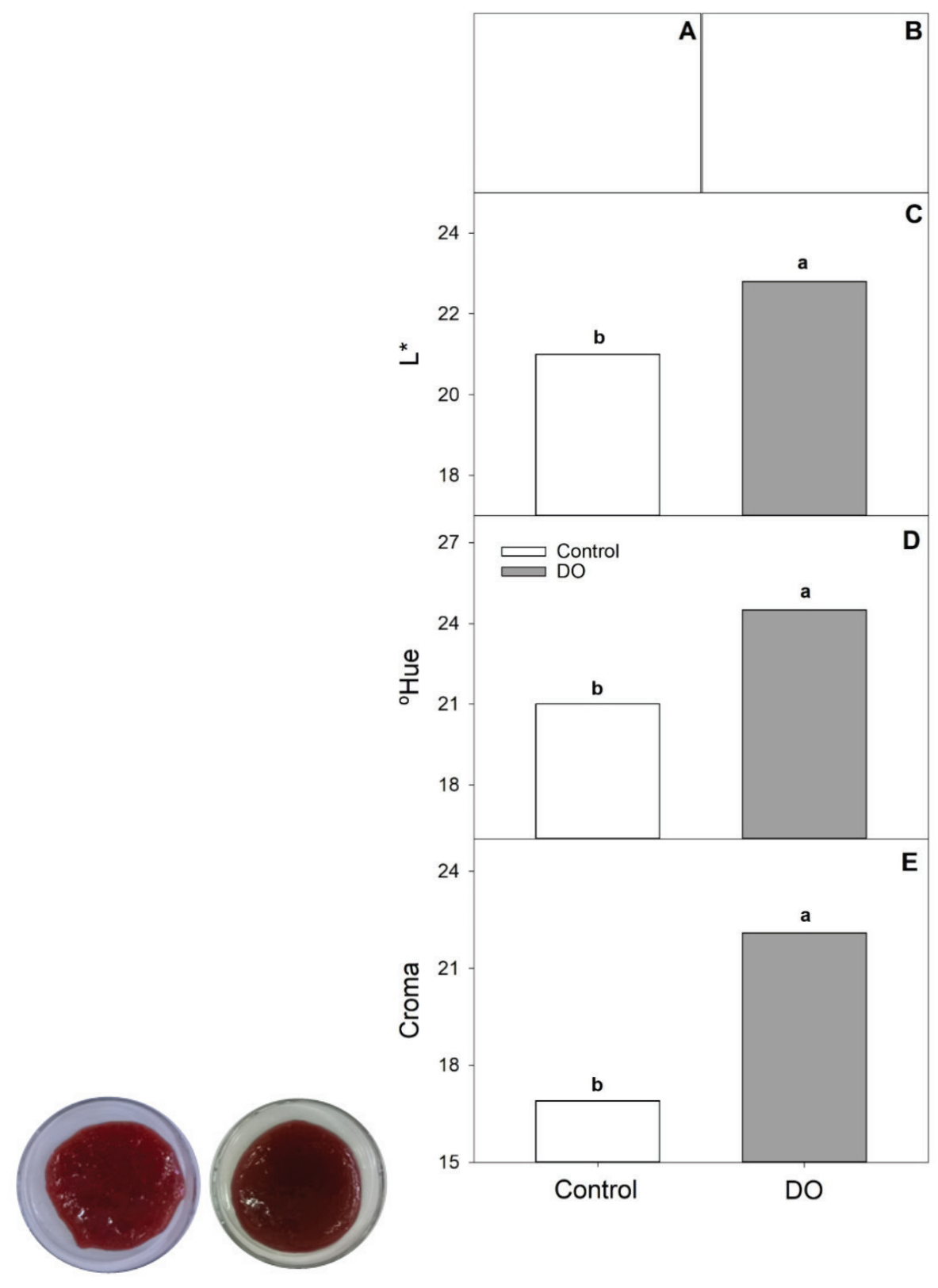

Figura 2: A, dulce de tomate elaborado de forma tradicional (Control); $B$, dulce de tomate elaborado con deshidratación osmótica previa (DO); C, luminosidad ( $\left.L^{*}\right) ; D$, ${ }^{\circ}$ Hue y $E$, Croma $\left(C^{*}\right)$, para los frutos control y DO. Diferentes letras indican diferencias significativas según un test de Tukey, $\alpha \leq 0,05, n=7$. 
Tabla 1: Valores de los parámetros de color $a^{*}$ y $b^{*}$ para los dulces de tomate elaborados de forma tradicional (control) y con deshidratación previa (DO). Diferentes letras indican diferencias significativas según un test de Tukey,

\begin{tabular}{ccc} 
& $\alpha \leq 0,05, n=7$. \\
\cline { 2 - 3 } & $\mathbf{a}^{*}$ & $\mathbf{b}^{*}$ \\
\hline Control & $15,8 \mathrm{~b}$ & $6,1 \mathrm{~b}$ \\
\hline $\mathbf{D} \mathbf{0}$ & $20,1 \mathrm{a}$ & $9,2 \mathrm{a}$ \\
\hline
\end{tabular}

\subsection{Contenido de Antocianinas y poder antioxidante} Luego del proceso de evaporación, los dulces con DO previa tuvieron un contenido de antocianinas de $183 \mathrm{mg}$ $\mathrm{kg}^{-1}$, mientras que las elaboraciones control rondaron los $153 \mathrm{mg} \mathrm{kg}^{-1}$ (Figura 3). El proceso de D0 extrae agua de los alimentos por diferencia de potencial osmótico entre la matriz del alimento y el osmolito altamente concentrado. Las antocianinas son compuestos solubles en agua. Transcurrido el proceso de D0, el 35\% de las antocianinas migró hacia el J (Figura 4), quedando las restantes 65\% en la pulpa. Luego de la concentración del jarabe J, el $40 \%$ del contenido de antocianinas se retuvo en el JC (13\% en relación al fruto fresco). Teniendo en cuenta que la mayor parte de las antocianinas fue retenida en la pulpa (FD), en principio podemos adjudicar el mayor contenido de antocianinas hallado en los dulces DO a un menor tiempo de exposición al calor (Figura 1). Hay trabajos que han sugerido que los azúcares pueden ejercer un efecto protector contra la degradación de las antocianinas (Watanabe et al., 2011). La mayor concentración de azúcares en la pulpa de las frutillas luego de la D0 podría haber estabilizado las antocianinas haciéndolas más tolerantes al procesamiento posterior (Barraza-Jáuregui et al., 2017).

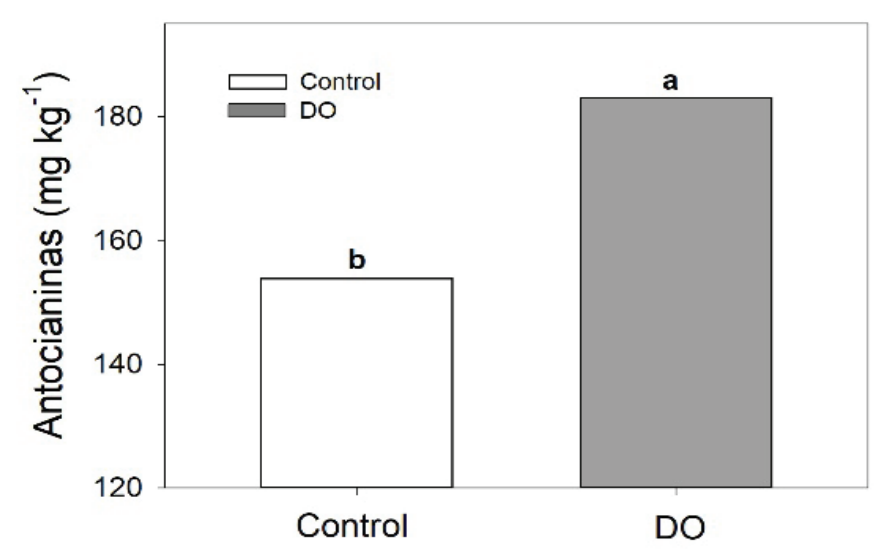

Figura 3: Contenido de antocianinas en dulces elaborados de forma tradicional (control) o con deshidratación osmótica previa (D0). Diferentes letras indican diferencias significativas según un test de Tukey, $\alpha \leq 0,05, n=7$. 


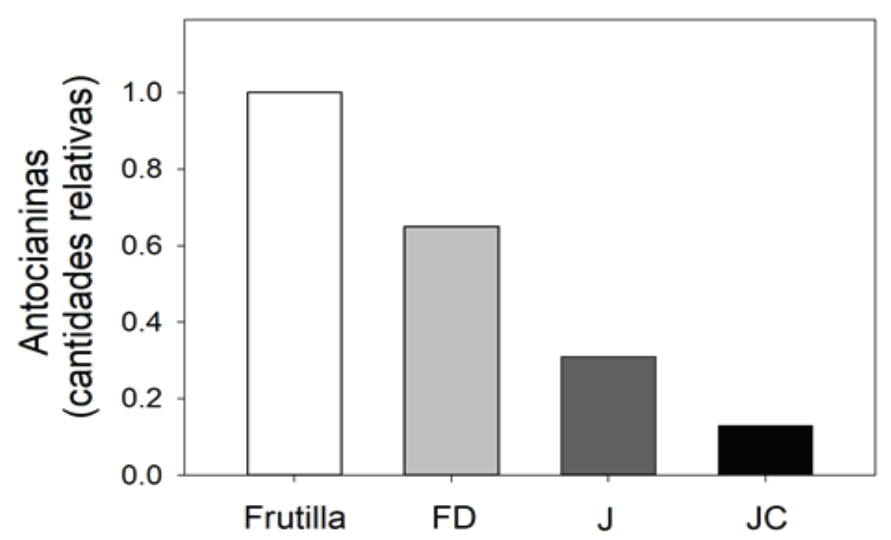

Figura 4: Cantidades relativas de antocianinas en dulces elaborados con deshidratación osmótica previa (DO). FD, fruta deshidratada; J, jarabe; JC, jarabe concentrado (ver figura 1).

La capacidad antioxidante contra el catión radical ABTS •+ fue significativamente mayor en los dulces con DO en comparación a los control (Figura 5). Se sabe que la degradación térmica de las antocianinas resulta en la generación de compuestos polifenólicos pero no está claro si esta degradación resulta en una pérdida neta de la capacidad antioxidante (Patras et al., 2010). De esta forma, el tratamiento térmico podría degradar las antocianinas convirtiéndolas en otros compuestos fenólicos que también posean actividad antioxidante. Por otra parte se conoce que algunos intermediarios de la reacción de Maillard generados durante los tratamientos térmicos pueden presentar capacidad antioxidante por lo que resulta necesario realizar un estudio más detallado.

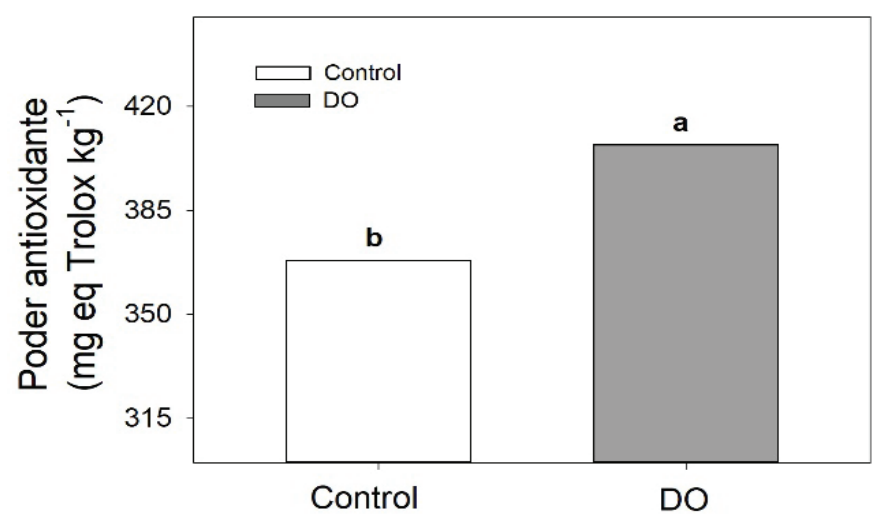

Figura 5: Poder antioxidante de dulces elaborados de forma tradicional (control) o con deshidratación osmótica previa (D0). Diferentes letras indican diferencias significativas según un test de Tukey, $\alpha \leq 0,05, n=7$.

\section{Conclusiones}

En este trabajo se elaboraron dulces de frutilla aplicando una deshidratación osmótica (DO), previa a la evaporación, utilizando el mismo azúcar de la formulación. Luego de la deshidratación osmótica, el $65 \%$ de las antocianinas quedó retenido en la pulpa. El proceso de D0 permitió reducir el tiempo de evaporación. Esto provocó que las antocianinas de los dulces con D0 estén menos expuestas al calor en comparación a los controles. Los dulces DO tuvieron mayor luminosidad, ${ }^{\circ}$ Hue y croma, con características más similares a la pulpa del fruto fresco que los obtenidos de forma tradicional. 
La diferencia en color podría estar relacionada con el mayor contenido de antocianinas hallada en los dulces DO. La capacidad antioxidante fue superior en los dulces DO. Esta información podría ser de utilidad para la industria de confituras.

\section{Referencias}

- Basu S, Shivhare US. (2013). Rheological, textural, microstructural, and sensory properties of sorbitol-substituted mango jam. Food and Bioprocess Technology, 6, 1401-1413.

- Barraza-Jáuregui G, Vega G, Valeriano J, Obregón J, Siche R, Miano AC. (2017). Osmotic pretreatment to assure retention of phenolics and anthocyanins in berry jams. Food Bioscience. 17, 24-28.

- Flores G, Del Castillo MLR. (2016). Cancer-related constituents of strawberry jam as compared with fresh fruit. Cancers, 8, 16.

- Jackman RL, Yada RY, Tung MA. (1987). A review: Separation and chemical properties of anthocyanins used for their qualitative and quantitative analysis. Journal of Food Biochemistry, .11, 279-308.

- Goulas V, Vicente AR, Manganaris GA. (2012). Structural diversity of anthocyanins in fruits. In: Motohashi $\mathrm{N}$. (Ed.), Anthocyanins: Structure, Biosynthesis and Health Benefits. Nova Science Publisherspp225-250.

- Holzwarth M, Korhummel S, Siekmann T, Carle R, Kammerer DR. (2013). Influence of different pectins, process and storage conditions on anthocyanin and colour retention in strawberry jams and spreads. LWT Food Science and Technology,. 52, 131-138

- Howard LR, Prior RL, Liyanage R, Lay J0. (2012). Processing and storage effect on berry polyphenols: challenges and implications for bioactive properties. Journal of Agricultural and Food Chemistry, 60, 66786693.

- Igual M, Sampedro F, Martínez-Navarrete N, Fan X. (2013). Combined osmodehydration and high pressure processing on the enzyme stability and antioxidant capacity of a grapefruit jam. Journal of Food
Engineering,.114, 514-521.

- Jain SK, Verma RC, Murdia LK, Jain HK, Sharma GP. (2010). Optimization of process parameters for osmotic dehydration of papaya cubes. Journal of Food Science and Technology,48, 211-217.

- Javanmard M, Chin NL, Mirhosseini SH, Endan J. (2012). Characteristics of gelling agent substituted fruit jam: studies on the textural, optical, physicochemical and sensory properties.International Journal of Food Science \& Technology, 47, 1808-1818.

- Levaj B, Bunić N, Dragović-Uzelac V, Kovačević DB. (2010). Gel Strength and Sensory Attributes of Fig (Ficus carica) Jams and Preserves as Influenced by ripeness. Journal of Food Science, 75, 120-124.

- Patras A, Brunton Nigel P, O'donnell C, Tiwari BK. (2010). Effect of thermal processing on anthocyanin stability in foods; mechanisms and kinetics of degradation. Trends in Food Science and Technology, 21, 3-11.

- Rababah Tm, Al-Mahasneh Ma, Kilani I, Yang W, Alhamad Mn, Ereifej K, Al-Údatt M. (2011). Effect of jam processing and storage on total phenolics, antioxidant activity, and anthocyanins of different fruits. Journal of the Science of Food and Agriculture, 91, 1096-1102.

- Rickman JC, Barrett DM, Bruhn CM. (2007). Nutritional comparison of fresh, frozen and canned fruits and vegetables. Part 1. Vitamins $C$ and $B$ and phenolic compounds. Journal of the Science of Food and Agriculture, 87, 930-944.

- Wang SY, Lewers KS. (2007). Antioxidant capacity and flavonoid content in wild strawberries. Journal of the American Society for Horticultural Science, 132, 629637.

- Watanabe Y, Yoshimoto K, Okada Y, Nomura M. (2011). Effect of impregnation using sucrose solution on stability of anthocyanin in strawberry jam. LWT - Food Science and Technology, 44, 891-895. 